\title{
Analysis of Retransmission Policies for Parallel Data Transmission
}

\author{
Imtiaz Ali Halepoto \\ Department of Computer Systems \\ Engineering, Quaid-e-Awam University \\ of Engineering, Science \& Technology, \\ Nawabshah, Pakistan \\ halepoto@quest.edu.pk
}

\author{
Intesab Hussain Sadhayo \\ Department of Telecommunication \\ Engineering, Quaid-e-Awam University \\ of Engineering, Science \& Technology, \\ Nawabshah, Pakistan \\ intesab@quest.edu.pk
}

\author{
Muhammad Sulleman Memon \\ Department of Computer Systems \\ Engineering, Quaid-e-Awam University \\ of Engineering, Science \& Technology, \\ Nawabshah, Pakistan \\ sulleman@quest.edu.pk
}

\author{
Adnan Manzoor \\ Department of Information Technology, Quaid-e-Awam \\ University of Engineering, Science \& Technology, \\ Nawabshah, Pakistan \\ adnan@quest.edu.pk
}

\author{
Shahid Bhatti \\ Department of Information Technology, Quaid-e-Awam \\ University of Engineering, Science \& Technology, \\ Nawabshah, Pakistan \\ shahidmsit12@gmail.com
}

\begin{abstract}
Stream control transmission protocol (SCTP) is a transport layer protocol, which is efficient, reliable, and connection-oriented as compared to transmission control protocol (TCP) and user datagram protocol (UDP). Additionally, SCTP has more innovative features like multihoming, multistreaming and unordered delivery. With multihoming, SCTP establishes multiple paths between a sender and receiver. However, it only uses the primary path for data transmission and the secondary path (or paths) for fault tolerance. Concurrent multipath transfer extension of SCTP (CMT-SCTP) allows a sender to transmit data in parallel over multiple paths, which increases the overall transmission throughput. Parallel data transmission is beneficial for higher data rates. Parallel transmission or connection is also good in services such as video streaming where if one connection is occupied with errors the transmission continues on alternate links. With parallel transmission, the unordered data packets arrival is very common at receiver. The receiver has to wait until the missing data packets arrive, causing performance degradation while using CMT-SCTP. In order to reduce the transmission delay at the receiver, CMT-SCTP uses intelligent retransmission polices to immediately retransmit the missing packets. The retransmission policies used by CMT-SCTP are RTX-SSTHRESH, RTXLOSSRATE and RTX-CWND. The main objective of this paper is the performance analysis of the retransmission policies. This paper evaluates RTX-SSTHRESH, RTX-LOSSRATE and RTXCWND. Simulations are performed on the Network Simulator 2. In the simulations with various scenarios and parameters, it is observed that the RTX-LOSSRATE is a suitable policy.
\end{abstract}

Keywords-CMT; CMT-SCTP; retransmission policies; SCTP; parallel transmission

\section{INTRODUCTION}

The most commonly used protocols of the transport layer of the OSI model are UDP and TCP. UDP is a connectionless and unreliable transport layer protocol. UDP sends data in the form of short messages, which are called datagrams, in a network. UDP is a connectionless protocol, which means that there is no need to establish a connection between the sender and receiver. In terms of reliability, the TCP is the most widely used protocol today. A recently introduced protocol for the transport layer is SCTP, which is reliable and connection-oriented. SCTP is very similar to TCP and UDP in terms of operations. It transmits multiple streams of data simultaneously between two endpoints that have established a connection. SCTP is more efficient and more powerful in its design when compared to peer protocols. CMT-SCTP extension enables a sender to simultaneously transmit data over various paths, which increase the overall transmission throughput. The competitor protocol to CMTSCTP is multipath TCP, which is also in design phase. Parallel transmission is very common in the development of mobile applications such as video streaming, online gaming, ecommerce, collaborative scientific projects and VoIP, that require faster data transmission and downloading data rate. The parallel transmission through CMT-SCTP uses more than one physical interface, for example the parallel transmission of data between two mobile phones uses two interfaces. One interface transmits using 4G and one interface transmits using WiFi. Sending data through two interfaces increases throughput compared to sending data through one interface. The use of more interfaces also increases the internet availability of a mobile phone. CMT-SCTP is in development process, and some issues such as transmission errors and recovery are challenging. The parallel transmission using CMT-SCTP causes unordered data packet arrival at the receiver. The data travels in parallel through different paths, the data along a fast path may reach the receiver earlier than the data sent along a slow path. To solve the problem an immediate retransmission of the missing data is mandatory for smooth transmission. In order to reduce the transmission delay at the receiver, CMT- 
SCTP utilizes some retransmission policies to quickly retransmit the missing data packets to the receiver. The main work of this paper is to evaluate retransmission policies for the performance analysis. There are five traditional retransmission policies of a CMT-SCTP sender. RTX-SAME and RTX-ASAP are very simple and outdated by the three newer policies. Obtained results show that RTX-LOSS RATE is better in terms of performance than RTX-CWND, and RTX-SSTHRESH. For the simulation setup a realistic network scenario is proposed. In the scenario, both paths with similar characteristics and dissimilar characteristics are tested. An example of dissimilar paths is parallel transmission using mobile phone via $4 \mathrm{G}$ and WiFi. To extend the simulation for more real networks, a loss rate is added in the paths to reflect the scenario of transmission errors. This research suggests the suitable retransmission policy as well as a base knowledge to design a new retransmission policy for CMT-SCTP.

\section{RETRANSMISSION POLICIES}

The retransmission policies used by CMT-SCTP are:

- RTX-SAME: In this retransmission policy data packet retransmission is expected to the similar node. It utilizes just a single path to send data packets to the same destination until that particular node is proved dead.

- RTX-ASAP: The sender retransmits missing data packets to a number of IP addresses. The receiver IP address that provides the congestion window (CWND) space is the retransmission address. If multiple destinations provide CWND space then the sender selects one randomly.

- RTX-CWND: In this policy the sender retransmits the data packets to the IP address with the largest CWND and to a random IP if CWNDs are equal.

- RTX-SSTHRESH: In this retransmission policy the sender is retransmitting data packets to the destination with the largest slow start threshold. In case of destinations with equal slow start threshold value, the IP address is chosen randomly.

- RTX-LOSSRATE: In this retransmission policy, the sender retransmits data a node with lowest path loss. Random selection is done in case of nodes with equal loss rates.

\section{SIMULATION MODEL}

Simulations have been carried out using Network Simulator 2 (NS-2) in which CMT-SCTP extension is available. The proposed scenario consists of one sender and one receiver. There are two paths used between the sender and receiver. In order to create these two paths the sender and the receiver are both configured as multihomed hosts. Each host is equipped with two interface cards. When the simulation begins the sender transmits the data in parallel along the two paths. The first step is setting up the NS-2 working parameters according to the proposed scenario. NS-2 has many default (fixed) parameters and many changeable parameters that are configured according to the proposed topology. In this research work, two scenarios are proposed as depicted in Table I. The first one includes paths of no packet loss. The second includes paths with packet loss. Four experiments are executed with the three CMT-SCTP retransmission policies. The simulation time is 300 seconds for each policy. The policies used are RTXSSTHRESH, RTX-CWND, and RTX-LOSSRATE. For each policy the simulation is repeated for 20 times and the average results are plotted. The protocol used is CMT-SCTP. Throughput is used as the evaluation parameter. The remaining parameters like the size of congestion window, buffer size at sender and receiver and the value of slow start threshold are set to the default settings of NS-2.

TABLE I. SIMULATION PARAMETERS

\begin{tabular}{|c|c|c|c|}
\hline \multicolumn{2}{|c|}{ Scenario without Loss Rate } & \multicolumn{2}{c|}{ Scenario with 10\% Loss Rate } \\
\hline$\#$ & Bandwidth, Delay & $\#$ & Bandwidth, Delay \\
\hline $\mathbf{1}$ & $\begin{array}{c}\text { Path } 1: 2-20 \mathrm{mbps}, 1 \mathrm{~ms} \\
\text { Path } 2: 20 \mathrm{mbps}, 1 \mathrm{~ms}\end{array}$ & $\mathbf{1}$ & $\begin{array}{c}\text { Path } 1: 2-20 \mathrm{mbps}, 1 \mathrm{~ms} \\
\text { Path } 2: 20 \mathrm{mbps}, 1 \mathrm{~ms}\end{array}$ \\
\hline $\mathbf{2}$ & $\begin{array}{c}\text { Path } 1: 22-40 \mathrm{mbps}, 1 \mathrm{~ms} \\
\text { Path } 2: 20 \mathrm{mbps}, 1 \mathrm{~ms}\end{array}$ & $\mathbf{2}$ & $\begin{array}{c}\text { Path } 1: 1-100 \mathrm{mbps}, 1 \mathrm{~ms} \\
\text { Path } 2: 1-100 \mathrm{mbps}, 1 \mathrm{~ms}\end{array}$ \\
$\mathbf{3}$ & $\begin{array}{c}\text { Path } 1: 5-50 \mathrm{mbps}, 200 \mathrm{~ms} \\
\text { Path } 2: 1 \mathrm{mbps}, 200 \mathrm{~ms}\end{array}$ & $\mathbf{3}$ & $\begin{array}{c}\text { Path } 1: 1 \mathrm{mbps}, 10- \\
100 \mathrm{~ms}\end{array}$ \\
\hline $\mathbf{4}$ & $\begin{array}{c}\text { Path } 1: 1 \mathrm{mbps}, 10-100 \mathrm{~ms} \\
\text { Path } 2: 1 \mathrm{mbps}, 1 \mathrm{~ms}\end{array}$ & $\mathbf{4}$ & $\begin{array}{c}\text { Path } 1: 1 \mathrm{mbps}, 1-100 \mathrm{~ms} \\
\text { Path } 2: 1 \mathrm{mbps}, 1 \mathrm{~ms}\end{array}$ \\
\hline
\end{tabular}

\section{RESUlTS \& DISCUSSION}

\section{A. Experiments Without Loss Rate}

The plots in Figure 1 show the performance of different retransmission policies executed without applying any kind of packet loss on the paths. Four scenarios are proposed with different bandwidth and delay values as shown in Table I. In the first scenario (Figure 1(a)) the policies are evaluated based on the bandwidth and the propagation delay changes. The bandwidth on path 2 or secondary path is kept to a fixed value, while the bandwidth on path 1 or primary path varies from 2 to $20 \mathrm{Mbps}$. The three policies behave very similar. Their starting performance is rather low from $2 \mathrm{Mbps}$ to $16 \mathrm{Mbps}$ and suddenly the throughput increases which is caused by the sudden increase in the congestion window. Figure 1(b) shows that there is a relationship between the bandwidth and the transmission throughput. In this experiment, a large value of bandwidth is used. In experiment 2 the retransmission rate of RTX-SSTHRESHOLD and RTX-CWND is very similar. However, due to the bandwidth availability (large bandwidth) the retransmission rate of RTX-LOSS rate is greater than the rates achieved with the other two policies. Experiment on a large bandwidth with longer delay values is performed in the scenario 3. It is observed that, when the delay is longer the impact of retransmission policy is less because the retransmission timer expires sooner no matter which retransmission policy is applied. The same is observed in experiment 4 , where a small bandwidth value is used and the delay value varies from $10 \mathrm{~ms}$ to $100 \mathrm{~ms}$ on path 1 . The experimentation with no loss rate shows that the bandwidth plays a key role in increasing the transmission throughput. The RTX-LOSSRATE policy improves the throughput with the increase in the bandwidth. On the other hand, propagation delay is a less significant parameter for the choice of retransmission policy. Moreover, the proposed scenario is very simple. 


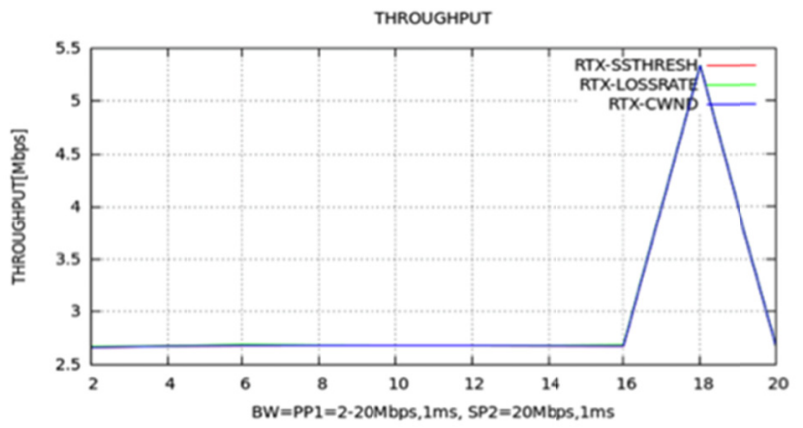

(a) Experiment 1: Bandwidth variation on path 1

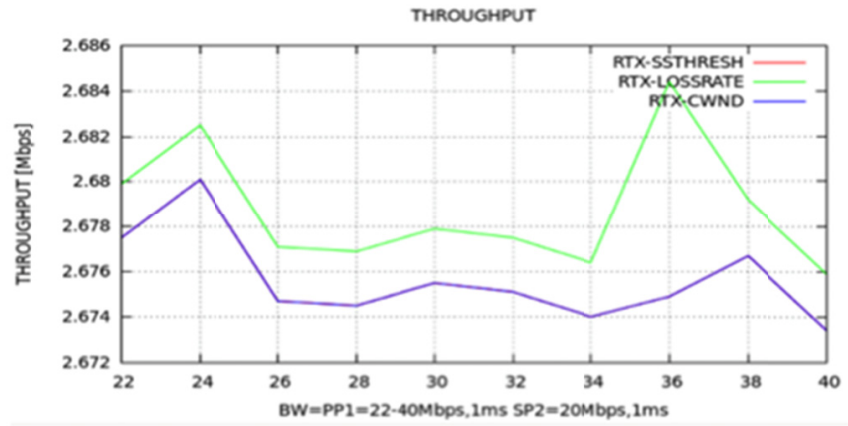

(b) Experiment 2: Large bandwidth

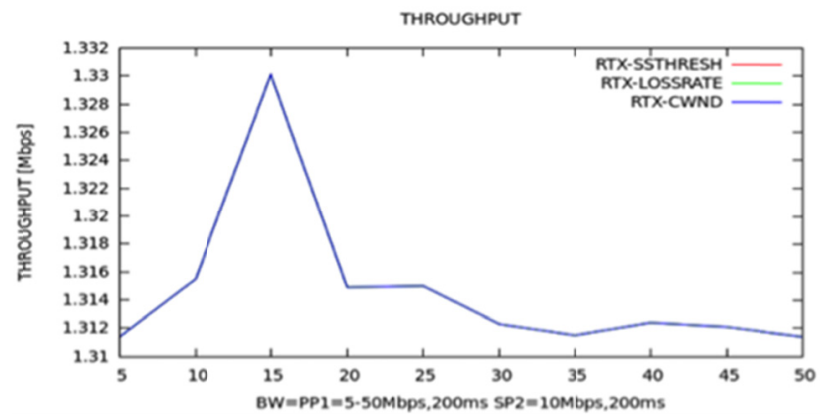

(c) Experiment 3: Delay variation on path 1

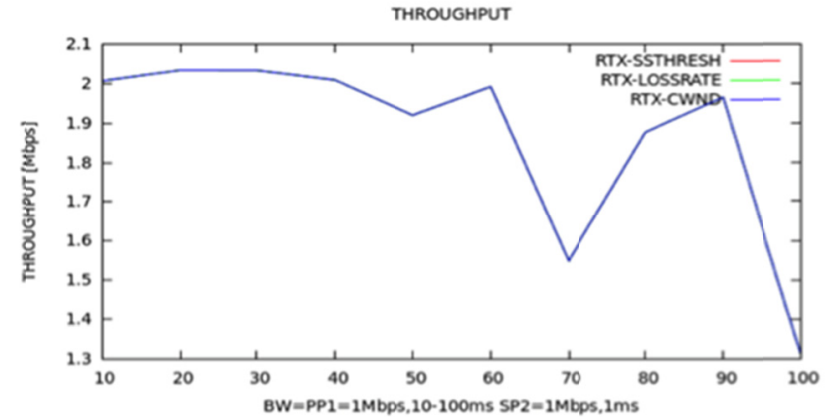

(d) Experiment 4: Small bandwidth with longer delay

Fig. 1. Scenario of two paths without loss rate

\section{B. Experiments With Loss Rate}

In reality, there are chances of data loss due to transmission errors. So, in this scenario the experiments are repeated with addition of programmed loss rate on Path 1 . The loss rate is configured to be $10 \%$. In experiment 1 , the bandwidth and delay on path 2 are kept $20 \mathrm{mbps}$ and $1 \mathrm{~ms}$. Whereas the bandwidth on path 1 changes from $2 \mathrm{Mbps}$ to $20 \mathrm{Mbps}$, however the delay remains constant on $1 \mathrm{~ms}$. When bandwidth is small between $2 \mathrm{Mbps}$ and $8 \mathrm{Mbps}$ there is no noticeable difference between the policies as show in Figure 2(a).

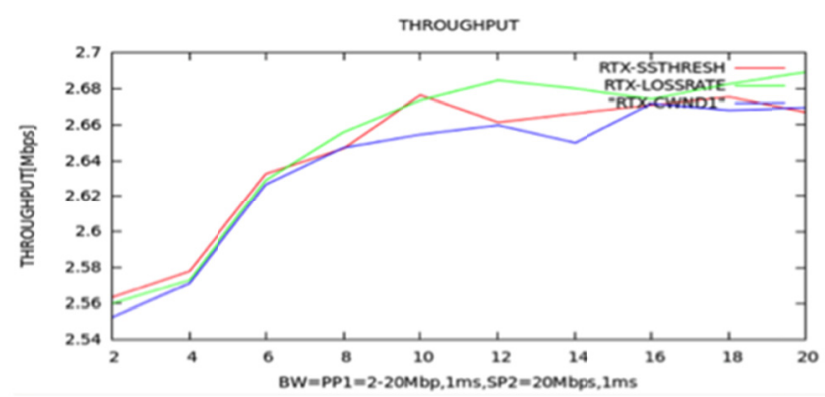

(a) Experiment 1: Bandwidth variation on path 1

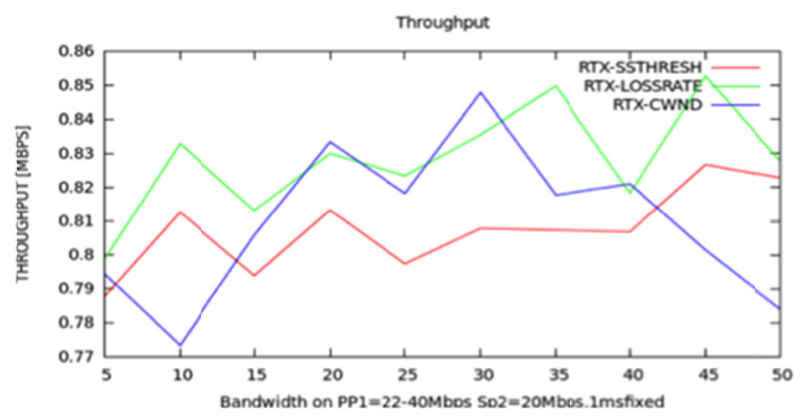

(b) Experiment 2: Bandwidth variation on both paths.

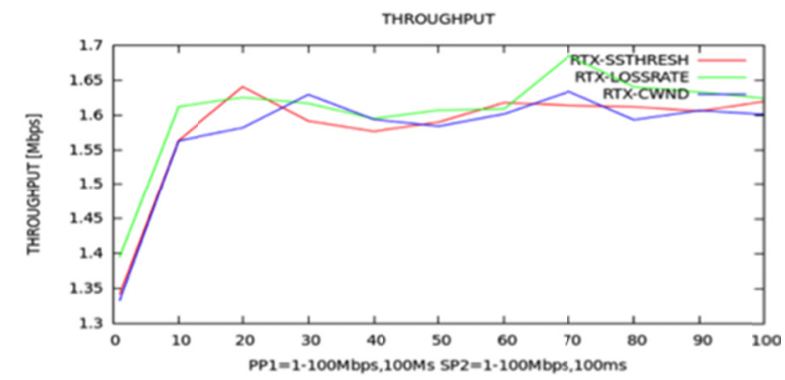

(c) Experiment 3: Bandwidth variation on both paths with longer delay

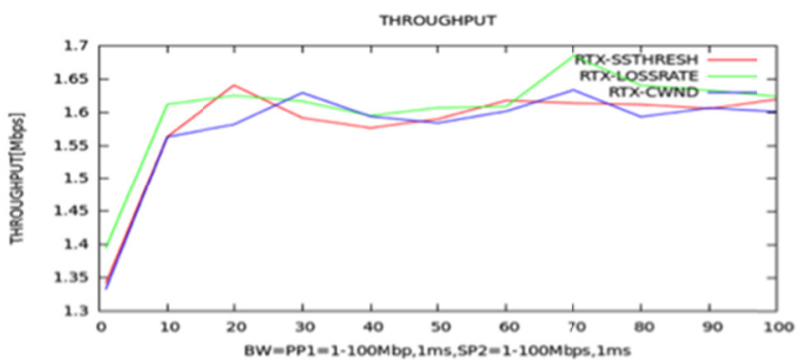

(d) Experiment 4: Bandwidth variation on both paths with larger bandwidth

Fig. 2. Scenario of two paths with added loss rate of $10 \%$ on path 1

When the bandwidth is larger than $8 \mathrm{Mbps}$, the RTXLOSSRATE reaches the throughput of $2.68 \mathrm{Mbps}$. The given experiment is slightly modified in experiment 2, where the bandwidth on path 1 increases from $22 \mathrm{Mbps}$ to $40 \mathrm{Mbps}$ (Figure 2(b)). In this experiment RTX-SSTHRESHOLD 
produces the lowest throughput. In the same experiment, the RTX-CWND, due to the large bandwidth the sender continuously increases the congestion window but due to the limitation in the buffer size of receiver the throughput at maximum reaches $0.84 \mathrm{Mpbs}$. The throughput of RTXLOSSRATE is greater than the other two policies. Experiment 3 (Figure 2(c)) is an example of two paths with similar characteristics i.e., same bandwidth and delay. The bandwidth on path 1 and 2 varies from $1 \mathrm{Mbps}$ to $100 \mathrm{Mbps}$. Longer delay is used i.e. $100 \mathrm{~ms}$. The trend in terms of throughput for the retransmission policies is the same. Initially with the increase in the bandwidth, the transmission rate also increases because at this point there are fewer chances of retransmissions and higher chances of successful transmissions due to the congestion window availability. In experiment 4 the bandwidth has a small value of $1 \mathrm{~ms}$ delay (Figure 2(d)). The results of experiment 4 are very similar to that of experiment 3 . This shows that on similar path characteristics the performance of all the retransmission policies remains similar. However, the experiment on paths with different characteristics, RTXLOSSRATE is the preferable policy for the data transmission.

\section{RELATED WORK}

Authors in [1] evaluated five retransmission polices of CMT-SCTP on a simple scenario where a sender transmits data to a receiver simultaneously through two paths. According to their observations RTX-LOSSRATE, RTX-CWND, RTXSSTHRESH are the best policies. They also suggested that in future, further work should be a new policy that must consider the loss rate into account $[2,4,5,11]$. The concept of integrated policy is proposed in $[3,10]$. The work in $[6,7,17]$ highlighted the role of the retransmission policy and suggested improvements in path selection while transmitting and retransmitting the data. Many authors evaluated SCTP and CMT-SCTP $[8,9,12]$ and highlighted that a good policy also reduces the buffer-blocking problem. The retransmission policies are also used for the evaluation of the CMT-SCTP extensions [13] along with other parameters. Authors in [14] related the importance of retransmission policies with the problem of handoff in wireless mobile networks. Authors in $[15,16]$ analyzed different failure scenarios of SCTP and their impact on the use of retransmission policies. The issue of retransmission or efficient transmission may also be solved by the design of routing protocols [18].

\section{CONCLUSION}

The trend of parallel transmission of data in order to obtain greater throughput is increasing. Parallel transmission is also useful in the development process of mobile applications for emergency services where a device is connected with more than one networks. One of the promising protocols for the parallel transmission is the CMT-SCTP. Transmission errors are common, particularly in parallel transmission where the immediate solution is the retransmission of data. For that, CMT-SCTP uses retransmission policies such as RTXLOSSRATE, RTX-CWND and RTX-SSTHRESHOLD. The current research compared the aforementioned retransmission policies in realistic network conditions where changes in bandwidth and delay are applied. Moreover, the programmed loss rate is introduced in the paths in the simulations. The results of simulations suggest that for similar path characteristics all of the retransmission policies behave the same. However, on the paths with added random data loss, RTX-LOSSRATE improves the throughput compared to RTXCWND and RTX-SSTHRESHOLD. The research may be extended to the evaluation of CMT-SCTP with other parallel transmission protocols such as multipath TCP.

\section{REFERENCES}

[1] J. R. Iyengar, P. D. Amer, R. Stewart, "Retransmission policies for concurrent multipath transfer using SCTP multihoming", 12th IEEE International Conference on Networks, Singapore, Vol. 2, pp. 713-719, IEEE, 2004

[2] J. R. Iyengar, P. D. Amer, R. Stewart, "Receive buffer blocking in concurrent multipath transfer", In IEEE Global Telecommunications Conference (GLOBECOM'05), St. Louis, USA, Vol. 1, p. 6, IEEE, 2005

[3] A. L. Caro, P. D. Amer, R. R. Stewart, "Retransmission schemes for end-to-end failover with transport layer multihoming", In IEEE Global Telecommunications Conference, GLOBECOM'04, Vol. 3, pp. 13411347, IEEE, 2004

[4] J. Liu, H. Zou, J. Dou, Y. Gao, "Reducing receive buffer blocking in concurrent multipath transfer", In 4th IEEE International Conference on Circuits and Systems for Communications, Shanghai, China, pp. 367371, IEEE, 2008

[5] P. Natarajan, N. Ekiz, P. D. Amer, R. Stewart, "Concurrent multipath transfer during path failure", Computer Communications, Vol. 32, No. 15, pp. 1577-1587, 2009

[6] I. A. Halepoto, F. C. Lau, Z. Niu, Z, "Concurrent multipath transfer under delay-based dissimilarity using SCTP”, In IEEE Second International Conference on Computing Technology and Information Management (ICCTIM), pp. 180-185, IEEE, 2015

[7] I. A. Halepoto, Scheduling and flow control in CMT-SCTP, HKU Theses Online (HKUTO), 2014

[8] P. Natarajan, J. R. Iyengar, P. D. Amer, R. Stewart, "Concurrent multipath transfer using transport layer multihoming: Performance under network failures", in Military Communications Conference, MILCOM 2006, Washington, DC, USA, pp. 1-7, IEEE, 2006

[9] J. R. Iyengar, P. D. Amer, R. Stewart, "Concurrent multipath transfer using transport layer multihoming: performance under varying bandwidth proportions", in IEEE Military Communications Conference, MILCOM 2004, Monterey, USA, Vol. 1, pp. 238-244, IEEE, 2004

[10] H. Shen, C. Wang, W. Ma, D. Zhang, "Research of the retransmission policy based on compound parameters in SCTP-CMT", in 2nd International Conference on Information Technology and Electronic Commerce (ICITEC), Dalian, China, pp. 25-28, IEEE, 2014

[11] A. L. Caro Jr, P. D. Amer, R. R. Stewart, "Retransmission policies for multihomed transport protocols", Computer Communications, Vol. 29, No.10, pp. 1798-1810, 2006

[12] T. Yang, L. Pan, L. Jian, H. Hongcheng, W. Jun, "Reducing receive buffer blocking in CMT based on SCTP using retransmission policy", in IEEE 3rd International Conference on Communication Software and Networks (ICCSN), Xi'an, China, pp. 122-125, IEEE, 2011

[13] Y. Cao, C. Xu, J. Guan, "A record-based retransmission policy on SCTP's Concurrent Multipath Transfer", in 2011 International Conference on Advanced Intelligence and Awareness Internet (AIAI 2011), Shenzhen, China, pp. 67-71, IEEE, 2011

[14] F. Siddiqui, S. Zeadally, "SCTP multihoming support for handoffs across heterogeneous networks", in: 4th Annual Communication Networks and Services Research Conference (CNSR 2006), Moncton, NB, Canada, IEEE, 2006

[15] A. L. Caro Jr, J. R. Iyengar, P. D. Amer, G. J. Heinz, R. R. Stewart, "Using SCTP multihoming for fault tolerance and load balancing", ACM SIGCOMM Computer Communication Review, Vol. 32, No.3, p. 23,2002 
[16] A. L. Caro Jr, P. D. Amer, J. R. Iyengar, R. R. Stewart, "Retransmission policies with transport layer multihoming", in: 11th IEEE International Conference on Networks, Sydney, Australia, pp. 255-260, IEEE, 2003

[17] I. A. Halepoto, F. C. M. Lau, Z. Niu, "Scheduling over dissimilar paths using CMT-SCTP", in: Seventh International Conference on Ubiquitous and Future Networks (ICUFN), Sapporo, Japan, pp. 535-540 IEEE, 2015

[18] N. H. Bhangwar, I. A. Halepoto, S. Khokhar, A. A. Laghari, "On routing protocols for high performance", Studies in Informatics and Control, Vol. 26, No. 4, pp. 441-448, 2017 